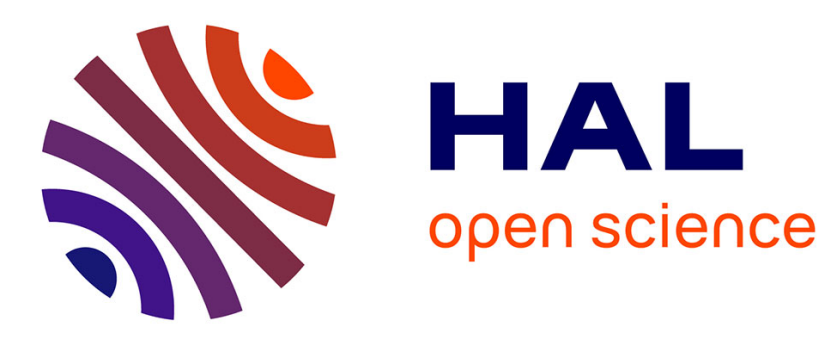

\title{
L'action publique en situation d'inachèvement. Que se passe-t-il quand ça s'arrête ?
}

Fabrizio Maccaglia, Hovig ter Minassian

\section{To cite this version:}

Fabrizio Maccaglia, Hovig ter Minassian. L'action publique en situation d'inachèvement. Que se passe-t-il quand ça s'arrête ?. Espace Géographique, 2014, 78 (2), pp.8-23. 10.3917/lig.782.0008 . hal-01081786

\section{HAL Id: hal-01081786 \\ https://hal.science/hal-01081786}

Submitted on 11 Nov 2014

HAL is a multi-disciplinary open access archive for the deposit and dissemination of scientific research documents, whether they are published or not. The documents may come from teaching and research institutions in France or abroad, or from public or private research centers.
L'archive ouverte pluridisciplinaire HAL, est destinée au dépôt et à la diffusion de documents scientifiques de niveau recherche, publiés ou non, émanant des établissements d'enseignement et de recherche français ou étrangers, des laboratoires publics ou privés. 


\section{L'action publique en situation d'inachèvement \\ Que se passe-t-il quand ça s'arrête ?}

\section{Fabrizio Maccaglia}

Université François Rabelais de Tours, laboratoire CITERES UMR 3724

fabriziomaccaglia@yahoo.fr

Université de Tours, 50 avenue Jean Portalis BP0607

37206 Tours Cedex 03

\section{Hovig Ter Minassian}

Université François Rabelais de Tours, laboratoire CITERES UMR 3724

hovig.terminassian@univ-tours.fr

Université de Tours, 50 avenue Jean Portalis BP0607

37206 Tours Cedex 03

\section{Résumé :}

Souvent stigmatisés comme symboles de la faillite d'un modèle politique ou économique, ou comme traces paysagères des magouilles ou de la corruption, les projets d'aménagement inachevés peuvent être aussi les supports de réappropriations artistiques ou symboliques. Ces initiatives, qui se sont particulièrement multipliées en Italie, nous invitent à rompre avec une lecture de l'inachèvement qui ne retient que les signes d'une pathologie ou d'un dysfonctionnement de l'action publique. Au contraire, elles montrent que l'inachèvement n'est pas la fin mais une étape transitoire dans l'histoire d'un projet. La notion d'inachèvement rend lisible à la fois les motifs qui peuvent expliquer l'échec, temporaire ou définitif, d'un projet d'aménagement ou d'une politique publique, mais aussi les possibles recyclages, revalorisations ultérieures symboliques, politiques ou marchandes, parfois longtemps après, de ce même projet ou de ce même territoire. Les projets inachevés continuent ainsi d'alimenter un imaginaire, d'influencer des initiatives, de susciter des politiques publiques et de produire des géographies.

Mots-clefs : inachèvement ; action publique ; appropriation ; mobilisation ; Italie.

\section{Summary:}

Often condemned as the icons of the failure of an economic or political model, or as the visual traces of fraud and corruption, unfinished urban planning projects can also support artistic or symbolical reappropriations. Those initiatives, which multiplied in Italy, lead to take distance with an interpretation of the unfinished which only consider them as the signs of a pathology or a dysfunction. On the contrary, those initiatives show that the unfinished is not the end but a transitory state in the history of an urban planning project. The idea of "unfinished" allows examining the causes of a failure, either temporary or definitive, of an urban planning project or of a policy, and in the same time studying the possible recycling, future symbolical, political or commercial revalorizations, sometimes many years later, of that project or policy. The unfinished continue to nourish the imaginary, to influence the local initiatives, to motivate policies and to produce territories.

Keywords: unfinished; public policies; appropriation; mobilization; Italy 
En 1961, Fidel Castro décidait la construction de l'École nationale d'Art de Cuba, à l'emplacement d'un ancien country club réservé aux élites avant la révolution de 1959. Cet établissement public avait pour vocation de proposer aux habitants de l'île des formations d'excellence à la danse, à la musique, aux arts plastiques et aux arts dramatiques. Le projet, confié à deux architectes italiens (Vitorrio Garatti et Roberto Gottardi) et à un architecte cubain (Ricardo Porro), suscita dès sa conception une controverse pour son style jugé par les détracteurs de l'époque comme trop maniériste, voire élitiste, ce qui ne cadrait pas avec les préceptes de la révolution castriste. La pénurie de matériaux, liée au blocus imposé par les Etats-Unis à partir de 1962 fut également en cause, si bien que le projet resta inachevé pendant près de quarante ans ${ }^{1}$. Ce n'est qu'en 2011 que les architectes du projet originel furent rappelés à Cuba pour achever leur œuvre. La même année, l'École nationale d'Art de Cuba était classée monument national.

Contrairement à ce que pourrait laisser entendre ce court exemple, les situations d'inachèvement ne sont pas propres aux pays en développement. En Italie, des documentaires (Unfinished Italy, Benoit Felici, 2011) leur sont consacrés ainsi que des circuits touristiques. Des collectifs d'artistes se sont emparés de la question pour interroger nos modèles de développement (économique, territorial) et notre rapport aux lieux et à la temporalité à travers ces étranges objets urbains ${ }^{2}$ que sont les portions d'autoroutes qui ne mènent nulle part (figure 1 : photo) ou ces grands stades sportifs inutilisés (à l'image du terrain de polo construit par la commune de Giarre en Sicile, alors même qu'il n'existe pas de fédération de polo dans l'île). Dans le cas de l'Espagne, le texte de Nacima Baron-Yellès dans le présent numéro rapporte quelques exemples d'artistes photographes ou vidéastes qui travaillent à partir de projets inachevés dans des perspectives similaires.

Compte tenu de l'importance atteinte par ces ouvrages «non finis », le législateur italien s'est récemment saisi de la question dans un contexte de crise économique où la programmation de nouvelles infrastructures est fortement corsetée par les contraintes budgétaires. L'article 44bis de la loi $\mathrm{n}^{\circ} 214$ du 22 décembre 2011 prévoit ainsi la création d'un registre national des équipements publics inachevés (Elenco-anagrafe nazionale delle opere pubbliche incompiute). Ce même article définit ce qu'est un équipement public inachevé, en reconnaissant quatre cas de figures d'inachèvement : 1) faute de crédits suffisants ; 2) suite à des contraintes techniques ; 3) consécutivement à l'introduction de nouvelles normes techniques ou réglementaires qui rendent caduques le projet ; 4) par manque d'intérêt de la part du commanditaire pour faire conclure les travaux. Les Régions et départements autonomes sont mandatés pour recenser les équipements inachevés disséminés sur leur territoire, en vue de les rendre opérationnels après avoir fait procéder à leur achèvement ou à leur adaptation.

À ce recensement institutionnel, qui s'inscrit dans une démarche d'action publique, s'ajoutent les initiatives portées par la société civile. La plateforme Incompiuto siciliano (www.incompiutosiciliano.org), définie comme un «observatoire public », donne à

\footnotetext{
${ }^{1}$ Le récit de ce projet est raconté dans le documentaire Unfinished Spaces (Alysa Nahmias, Ben Murray, 2011).

${ }^{2}$ Le collectif Alterazioni video définit en ces termes le projet Incompiuto siciliano qu'il anime : « Le travail de recherche sur l'architecture inachevée porte sur les équipements publics inachevés en Italie. Nous questionnons, selon une approche pluridisciplinaire, les relations entre ces œuvres architecturales et le contexte dans lequel elles s'inscrivent, en en rappelant leur valeur et en en proposant une nouvelle définition stylistique. Nous regardons l'ensemble du "système national des équipements inachevés", dont nous avons réalisé le recensement en quelque deux années de recherche, comme un témoignage matériel du contexte socio-culturel contemporain qui imprègne notre quotidienneté, et nous proposons une définition de ce nouveau style qui en restitue les contenus à partir d'une multitude de points de vue ». Pour la France, on regardera par exemple les travaux du collectif Coloco.
} 
quiconque la possibilité de signaler la présence d'un équipement inachevé. La base de données en dénombrait 320 en janvier $2012^{3}$. La plateforme (Im)possible living (www.impossibleliving.com), qui a pour slogan Improve your city. Share your energy to reactivate abandoned buildings, est quant à elle un espace collaboratif qui invite les visiteurs du site à soumettre des projets de reconversion ou de réutilisation (figures 2 et 3 : captures d'écran). Chaque équipement recensé est accompagné d'une fiche signalétique précisant sa localisation, l'environnement dans lequel il s'inscrit et son histoire, ainsi que ses caractéristiques techniques et matérielles. Ce matériau est mis à disposition de tous, professionnels (architectes, urbanistes, artistes) comme non professionnels, pour ouvrir un concours de projets permanents ou éphémères (happenings, installations artistiques, etc.). Il s'agit d'encourager la réhabilitation ou la réutilisation, et rompre ainsi avec une logique consumériste du territoire en associant développement durable, innovation technologique et mobilisation citoyenne (l'objectif étant d'impulser des initiatives locales à l'échelle mondiale).

Si les artistes sont interpellés par les projets inachevés, les chercheurs en sciences sociales peuvent l'être également ${ }^{4}$. En général, les situations de blocage d'un projet ou d'une politique publique sont étudiées sous l'angle des rapports de force géopolitiques et des situations de conflits (Subra, 2007 ; Melé, 2008), des mobilisations à leur encontre (Fourniau, De Donzel, 2001 ; Fourniau, 2007 ; Dupuy, Halpern, 2009 ; Melé, 2013), ou de leurs effets sur le projet (Lolive, 1997). Avec la notion d'inachèvement, nous nous proposons de déplacer la focale, considérant que l'inachèvement n'est pas la fin mais une étape transitoire dans l'histoire d'un projet. Elle rend lisible à la fois les motifs qui peuvent expliquer l'échec, temporaire ou définitif, d'un projet d'aménagement ou d'une politique publique, mais aussi les possibles revalorisations ultérieures symboliques, politiques ou marchandes, parfois longtemps après, de ce même projet ou de ce même territoire. Ici, il s'agit donc de regarder non (seulement) le passé du projet ou de la politique publique, en décryptant pourquoi il reste inachevé mais (aussi) son futur : que devient-il par la suite?

\section{De l'achèvement à l'inachèvement}

Pour comprendre l'inachèvement, il nous faut d'abord comprendre l'achèvement. À quelles conditions peut-on dire qu'un projet d'aménagement ou qu'une politique publique est achevée ? Nous opérons pour cela un petit détour par les théories de l'action publique ${ }^{5}$, en nous basant sur les propositions de Pierre Hassenteufel (2011). Trois hypothèses sont alors possibles pour comprendre l'achèvement d'une politique publique.

La première hypothèse postule qu'une politique publique se termine lorsque le problème dont les autorités se sont saisies est résolu. Cela suppose que soit clairement défini, que les objectifs soient énoncés clairement, que les résultats soient mesurables et que le problème traité soit soluble. Ces conditions sont rarement réunies dans le cadre d'une politique publique, qui le plus souvent traite des problèmes multidimensionnels et aux contours flous, avec des objectifs multiples voire contradictoires, et qui porte sur des problèmes récurrents

\footnotetext{
${ }^{3}$ Jacopo Fo, «Opere pubbliche, le 320 incompiute », Il fatto quotidiano, 30012012.

${ }^{4}$ Cette publication fait suite à une journée d'études organisée le 25 janvier 2013 par l'équipe CoST du laboratoire CITERES (UMR 7324), sur le thème «Fermé pour travaux. L'action publique saisie au travers et l'inachèvement». Nous remercions ici Christophe Demazière et Patrice Melé pour leur contribution à l'animation des débats.

5 À l'origine, la journée d'études sur l'inachèvement portait plutôt sur des cas de projets d'aménagement ou de politiques publiques. Les débats qui s'y sont tenus ont permis d'élargir plus généralement la discussion à l'action publique, défini comme «l'activité des pouvoirs publics et plus largement toute activité articulée sur un espace public et nécessitant une référence à un bien commun » (Laborier, Trom, 2003).
} 
difficiles à traiter (comme la pauvreté, le chômage, la criminalité, la protection de l'environnement...). Il est parfois d'autant plus difficile de considérer une politique publique comme achevée dès lors que cette politique peut avoir, comme le rappellent Mény et Thoenig (1989), des objectifs qui ne sont pas nécessairement explicités, donc aisément évaluables. Quels sont alors les critères possibles pour estimer le degré d'achèvement ou d'accomplissement d'un politique publique? Le seuil des $20 \%$ de logements sociaux dans une commune suffit-il pour considérer qu'une politique de logement social est achevée, ou ne faut-il pas au contraire considérer que l'évaluation numérique du nombre de logements sociaux produits n'est qu'une des facettes de la question?

La seconde hypothèse postule qu'une politique publique est achevée lorsqu'une décision est effectivement appliquée. 'une part, il existe des distorsions entre la décision et sa mise en œuvre : plus il y a d'échelons administratifs entre le niveau d'élaboration de la décision et celui de son application, plus la décision peut subir des altérations (du fait des jeux de concurrences entre administrations). Elle subit également une interprétation de la part des agents de terrain chargés de l'appliquer (les street level bureaucrats), et les publics ciblés par l'intervention peuvent réagir autrement de ce qui est attendu. D'autre part, il faut prendre en compte la dynamique temporelle d'une politique publique. $\mathrm{n}$ projet peut rester longtemps inachevé pour ressortir, un jour, des cartons, comme dans le cas de l'École nationale d'Art de Cuba. Dans son intervention sur les politiques urbaines à Bucarest lors de la journée d'étude sur l'inachèvement, Samuel Rufat proposait, dans ces conditions, de parler de cyclicité plutôt que d'inachèvement, pour évoquer la réactivation de projets parfois anciens ou de stratégies de développement urbain qui peuvent être pensés en termes de continuité ou au contraire de rupture avec la période précédente. Il s'appuie notamment sur la volonté récurrente depuis le $19^{\text {ème }}$ siècle de faire de Bucarest une véritable «ville européenne » et les politiques urbaines afférentes, mais aussi sur l'exemple du Centre Civique de Bucarest, projeté dans les années 1930, devenue Palais du Peuple durant la période communiste, avant d'accueillir le siège du Parlement depuis 1989. C'est bien l'ensemble des politiques urbaines de la capitale roumaine qui peut ainsi se lire sous l'angle de ces logiques de rupture avec le passé et de reconstruction symbolique : «deux préoccupations se font récurrentes [depuis le $19^{\text {ème }}$ siècle], d'une part « urbaniser » la capitale, d'autre part, la doter de monuments, d'un centre capable de supporter la comparaison avec les autres capitales européennes. Toutefois, il semble qu'à chaque fois les constructions nouvelles, qui se veulent en rupture avec le passé, se greffent malgré l'ampleur des destructions sur les structures antérieures, par un retour des projets et des pratiques, sans réellement parvenir à faire une ville nouvelle » (Rufat, 2008, p. 53). Enfin, la troisième hypothèse de Pierre Hassenteufel est que l'on peut considérer qu'une politique publique est considérée comme achevée lorsque les unités administratives chargées de sa mise en œuvre sont vouées à disparaître. En réalité, selon l'auteur, cette situation est relativement rare, puisque même si l'échelon administratif disparaît, ses missions, notamment en matière de politiques publiques, sont en général transférées à d'autres structures ou d'autres entités administratives. L'expérience montre que la tendance est plutôt à la résistance des structures administratives et des publics bénéficiant des politiques publiques.

Ces éléments nous montrent qu'une politique publique continue à produire des effets audelà de son application stricto sensu: une politique publique n'a pas de fin (ou très rarement); même lorsqu'elle n'existe plus «officiellement » elle est recyclée dans d'autres programmes.

\section{Les figures de l'inachèvement}


A présent qu'entend-on par inachèvement? L'inachèvement renvoie à un état, à un moment donné, au sujet duquel on peut faire le constat d'un manque, d'une absence : l'équipement planifié ne possède pas les propriétés qu'ils devraient, pourtant, posséder, ou bien les politiques publiques n'ont pas atteint les objectifs fixés. De fait, une part d'incertitude accompagne tout projet d'aménagement liée aux obstacles, aux difficultés, à l'imprévu qui peuvent surgir tout au long du processus qui débute avec la conception du projet jusqu'à la livraison du chantier. Il arrive parfois que le projet avorte avant même que les travaux ne commencent que le projet débute puis s'enlise, s'arrête en cours de route : erreurs de conception, sous-estimation des coûts, faillite des entreprises en charge des travaux, recours et contre-recours judiciaires, épuisement des sources de financement, changement de majorité politique... autant de raisons qui peuvent entraîner une interruption des travaux. Il reste alors un équipement inachevé, appelé à devenir une friche si le chantier n'est pas relancé. Même inachevé, un équipement est cependant capable de modeler le paysage et de donner lieu à des formes d'appropriation sociale ou de mobilisation collective, comme le souligne le texte de Philippe Bachimon dans le présent numéro ; même inachevé, un équipement peut produire des effets sociaux. Les équipements inachevés ne peuvent pas non plus être considérés comme des friches qui subissent une « (...) désaffection consécutive à des changements de modes de production et d'organisation sociale »(Veschambre, 2005, p. 86). N'ayant jamais fonctionné ils ont échappé à toute forme de déclassement ou de péremption; leur état a, en quelque sorte, été suspendu, figé, jusqu'à ce qu'un processus de recyclage et de réappropriation s'enclenche (Veschambre, 2005).

Étudier l'inachèvement dans le domaine de l'aménagement ou de l'urbanisme, c'est alors tenter de croiser deux interrogations : d'une part la question de l'évaluation des politiques publiques, d'autre part celle de l'appropriabilité des territoires ou des projets considérés comme en friches, inachevés. Cette question est notamment au cœur du texte de Philippe Bachimon dans le présent numéro. Est inachevé ce qui ne remplit pas les objectifs fixés au départ du projet (d'aménagement ou de politique urbaine). On ira même jusqu'à affirmer qu'un équipement ou un projet inachevé est un objet «virtuel », le terme étant entendu ici non au sens d'inexistant, mais au sens deleuzien du terme : est virtuel ce qui est en puissance (du latin vis, signifiant puissance) (Lévy, 1998). Par son état inachevé, le projet ou l'équipement considéré ouvre le champ à des possibles réappropriations, réutilisations ou recyclages. Donc, plus un projet est éloigné de son état d'achèvement, plus il est virtuel : cela ne signifie pas qu'il existe « moins », mais au contraire qu'il offre une plus grande marge de manœuvre à des réappropriations multiples.

Cette caractéristique du projet inachevé comme objet virtuel peut parfois contaminer certains projets urbains dont les usages ne sont pas toujours définitivement actés a priori. Cette dernière proposition fait écho à l'analyse que l'on trouve chez Hervé Gumuchian (1991), qui distingue au moins trois stratégies différentes de production de l'espace, selon l'articulation qu'elles offrent entre projet d'aménagement et discours sur l'espace :

- Les stratégies de prescription: par ces stratégies, les acteurs publics cherchent à structurer l'espace de vie des usagers ou des riverains par des aménagements qui contraignent plus ou moins fortement les mobilités ou les pratiques du lieu, selon des objectifs explicites : le cas d'une galerie marchande ou d'une zone d'aménagement commercial, pensés pour certains types d'usages à l'exclusion d'autres, mais c'est aussi le cas d'une voie rapide, avec un nombre nécessairement limité d'entrées et de sorties, et en général interdite aux modes de déplacements autres que l'automobile ;

- Les stratégies de séduction ou de suggestion (ou de prescription implicite) : par cellesci, l'usager est amené, par incitation, à certains comportements spatiaux ou certains types d'usage. Moins coercitives que les premières, elles n'induisent pas moins des 
modalités circonscrites d'appropriation de l'espace, par exemple par des chemins balisés pour des randonnées pédestres, des itinéraires cyclables, l'aménagement de voies piétonnes (pour inciter à la flânerie). Ces projets imposent, dans son organisation et son utilisation fonctionnelle, des usages ou des besoins préalablement identifiés, tout en soutenant un discours affirmant la multiplicité des fonctions ou des modes de déplacement ;

- Enfin, les stratégie de laisser-faire, ou de permission : dans ces dernières, les espaces sont conçus dès l'origine par les acteurs de l'aménagement comme ouverts, multifonctionnels et susceptibles d'être appropriés de différentes manières par les usagers. Il s'agit alors de laisser aux riverains, aux populations locales, la possibilité de se réapproprier un équipement ou un espace public comme ils le souhaitent. Le parvis aménagé devant le musée d'art contemporain de Barcelone (MACBA), inauguré en 1995, est un bon exemple de ce type d'aménagement (Sokoloff, 1999).

Entre un projet inachevé et un projet terminé aux objectifs peu marqués, il n'y aurait donc substantiellement pas de différence fondamentale.

À l'inverse, l'inachèvement n'est pas synonyme de ruine. La ruine est « (...) un vestige, un fragment, finalement une trace qui renvoient le voyageur au souvenir du passé » (Fournet, 2007, p. 23). L'idée de ruine suppose l'usure du temps, l'altération de la forme, le délabrement ; elle renvoie à une existence passée, à ce qui fut et n'est plus, mais continue malgré tout d'exister au travers de traces parfois fugitives à l'image de ces sites de l'antiquité où des monuments ne subsiste qu'un tapis de pierres. La ruine invite à la méditation, à la contemplation, à une forme d'abandon: elle est «l'exemple d'une architecture qui ne fonctionne plus mais qui pourtant continue à communiquer avec la même intensité et avec la même présence » (Bello Marcano, 2013, p. 7). La ruine nous inscrit dans une temporalité, voire une filiation culturelle lorsque nous y projetons nos racines. La relation à la ruine est enfin fortement empreinte d'esthétisme au-delà de sa valeur historique.

Tout autre est la relation à l'inachevé car celui-ci, loin de susciter le ravissement, nourrit des sentiments de rejet et d'indignation (à l'encontre de ces hommes incapables de porter à terme leur œuvre ou des pouvoirs publics en faillite). Faute d'existence, le temps ne peut pas, comme dans le cas de la ruine, rappeler l'objet que l'inachevé a été. Le voyageur se détourne de cet équipement privé de dignité, un peu comme dans un processus de refoulement. C'est donc un imaginaire qui fait la part belle au ressentiment et à l'oubli qui est communément associé à l'inachevé comme en témoignent les réactions des habitants de la commune de Giarre interrogés par Benoît Felici dans son film (Unfinished Italy). C'est dans ce contexte que s'inscrit le manifeste de l'Incompiuto siciliano: l'Inachevé sicilien est un projet «de relecture du paysage italien, qui vise à renverser la perception négative que nous avons des équipements publics inachevés jusqu'à en déclarer la dignité d'œuvre d'art et de les transformer ainsi en ressource économique ». L'inachèvement sicilien est théorisé : c'est un style architectural à part entière. C'est le " paradigme interprétatif de l'architecture italienne de l'après-guerre à nos jours ». Les équipements inachevés n'existent pas isolément les uns des autres; ils font système car, produits en série, ils s'inscrivent dans la même séquence historique où le politique et les milieux d'affaires se sont mutuellement construits, tant au niveau local que national, par le biais d'échanges corruptifs et de transactions informelles (Maccaglia, 2013).

Ruine et inachevé sont, cependant, l'un et l'autre une invitation. Dans un cas à se souvenir, souvent d'un passé merveilleux (la ruine est ainsi le support d'une démarche mémorielle), dans l'autre à imaginer le futur, à agir, à faire (l'inachevé est le support d'un engagement). La ruine induit également un traitement spécifique : l'abandon lorsque sa valeur 
patrimoniale ou historique est faible ; la restauration ou la conservation dans le cas inverse. L'inachevé induit une toute autre démarche : compléter pour utiliser ; transformer pour tirer profit. On s'inscrit à contre-courant d'une tendance où «le recyclage par la démolition demeure le principal levier de transformation urbaines »(Veschambre, 2005, p. 82). Des artistes et des professionnels prennent cependant leurs distances avec cette approche utilitariste pour qui l'inachevé doit être laissé en l'état.

\section{Penser l’inachèvement pour penser les réappropriations}

L'inachèvement permet donc de penser différemment l'action publique ou les projets d'aménagement: non pas en termes de réussite/échec, mais en termes de recyclage, de glissement de sens, de réappropriation ou de réactivation. Nous proposons ici deux exemples pour l'illustrer.

D'abord, du côté des politiques publiques, on peut prendre le cas des politiques de «gentrification», ce processus étant défini comme «une forme particulière d'embourgeoisement des quartiers populaires qui passe par la transformation de l'habitat, voire de l'espace public et des commerces » (Clerval, 2010). Pour les chercheurs spécialistes de la question, l'une des questions est de savoir si les contextes de crise économique ou immobilière peuvent marquer un coup d'arrêt aux processus de gentrification. S'intéressant aux métropoles canadiennes dans le contexte de la récession économique du tournant des années 1980 et 1990, L. Bourne montre ainsi que la gentrification a concerné un nombre limité de quartiers des villes-centres, en particulier à Toronto (1993). Il avance l'idée que la gentrification dans les métropoles nord-américaines n'a pas abouti, et que celles-ci entrent alors dans une ère de post-gentrification, caractérisée, entre autres, par la baisse des prix immobiliers, la précarisation de l'emploi et sa croissance dans les territoires suburbains. Certes dans les années 1990, la gentrification a marqué le pas dans le quartier londonien d'Islington comme dans le Lower East Side, à New York (Lees, Bondi, 1995), mais pour un temps seulement. Loin de la post-gentrification prônée par L. Bourne, les années 1990 sont plutôt la décennie d'une gentrification internationalisée et généralisée (Carpenter, Lees, 1995 ; Smith, 1996 et 2002). Dans le cas de New York, bien étudié, la crise fiscale des années 1973-1977 a entrâné le désengagement de la municipalité et la mise en place de systèmes fiscaux incitatifs qui ont favorisé l'afflux de capitaux privés en direction des marchés dévalorisés (Smith, 2003). Ainsi, la gentrification, en tant que phase de recomposition sociale et urbaine des quartiers anciens, aurait déjà traversé trois crises économiques avant d'affronter la crise de la fin des années 2000 (Lees, 2009). Selon N. Smith, une crise économique n'arrête pas la gentrification, bien au contraire. En limitant la marge de manœuvre des municipalités, en modifiant les contraintes économiques qui pèsent sur les politiques d'aménagement et en encourageant le financement du logement par le secteur privé, les crises économiques favorisent le passage de la gentrification comme processus de transformation sociale en «stratégie urbaine globale » (Smith, 2003, p. 60). L. Lees est plus mesurée : elle évoque plutôt un filtrage des quartiers en cours de gentrification : en bref, ce sont dans les quartiers où le processus est le mieux consolidé que ce dernier résisterait le mieux, étant les plus attractifs, les autres expérimentant plutôt une dégentrification, c'est-à-dire un reflux du processus (Lees, 2009). Cette discussion montre toute la difficulté à anticiper à court ou moyen terme sur les dynamiques sociales d'un quartier ou d'une métropole. Parce que le processus de gentrification, et les politiques qui la soutiennent, en particulier en Amérique du Nord, sont pensés en termes de réussite, d'aboutissement ou d'échec, ces réflexions ne laissent pas la place à l'hypothèse de possibles reflux ou au contraire de réactivations de certaines dynamiques sociales. Penser les politiques de gentrification en termes 
d'achèvement/inachèvement permet au contraire de reporter l'attention sur ce qui, un temps, peut ralentir ou au contraire accélérer la transformation sociale des villes, mais aussi de sortir d'une logique d'inéluctabilité de facteurs micro ou macro-économiques.

Ensuite du côté des projets urbains, on peut prendre l'exemple du centre historique de Palerme. Les pouvoirs publics ont, au lendemain du econd conflit mondial, donné la priorité à l'urbanisation des périphéries dans un contexte de forte croissance démographique. Palerme, qui accède au rang de capitale régionale en 1947, voit affluer des milliers d'habitants qui abandonnent leurs campagnes pour trouver un emploi urbain. La rentabilité des opérations immobilières en périphérie dans un contexte fortement spéculatif conduit à reporter les interventions urbanistiques projetées dans le centre historique, notamment le percement d'un axe pour faciliter la circulation automobile et la substitution du bâti existant pour partie composé de palais aristocratiques hérités des $\mathrm{XVII}^{\mathrm{e}}$ et $\mathrm{XVIII}^{\mathrm{e}}$ siècle par des immeubles collectifs d'habitation à l'image de ceux en cours de construction. La fin des années 1970 et le début des années 1980 marquent un essoufflement de l'urbanisation palermitaine. La municipalité ne peut plus arguer de la crise du logement pour justifier les opérations immobilières dans le centre historique compte tenu de la stabilisation de la croissance démographique et des premiers signes de desserrement urbain. Si une demande de logement social subsiste de la part des franges les plus modestes de la société locale, cette demande n'a nullement vocation à être satisfaite en centre-ville et par des investisseurs privés. Le contexte politique a également changé, limitant les interventions non maîtrisées ou spéculatives. L'idée de la préservation et de la réhabilitation du centre historique s'impose progressivement parmi les élites urbaines, et se concrétise dans l'adoption d'un plan d'urbanisme spécifique au centre-ville en vue d'en assurer la requalification (1986). Cet effet de conjoncture débouche au final sur un paradoxe : on lui doit à la fois l'état de fort délabrement dans lequel se trouve le centre historique (plusieurs immeuble se sont effondrés au cours des dernières années) et en même temps sa préservation. Celle-ci est directement imputable à l'impossibilité de l'administration palermitaine de conduire à terme la politique urbaine projetée dans les années 1950.

L'inachèvement permet de penser les appropriations et réappropriations possibles d'un projet ou d'un territoire.

L'appropriation est l'expression de la capacité d'un groupe social à occuper de manière plus ou moins exclusive un territoire (Ripoll, Veschambre, 2005). L'appropriation a d'abord une dimension juridique, celle du droit de propriété, mais elle ne s'aurait s'y limiter. Certes, la question de la propriété est essentielle puisqu'elle renvoie au statut d'un terrain ou d'un logement, aux opportunités d'occupation et plus généralement à l'inégalité des ressources entres différentes catégories sociales de la population. Mais l'appropriation a aussi une dimension plus symbolique, liée aux usages, notamment à ceux des espaces publics, et aux pratiques culturelles ou commerciales auxquels les différents groupes sociaux ont plus ou moins accès sur un même territoire.

Unfinished Italy fait ressortir le caractère discursif ou symbolique de l'appropriation (Di Meo, 2007) : au-delà de son aspect matériel (lorsqu'il s'agit d'un monument) ou immatériel (lorsqu'il s'agit d'une pratique culturelle), l'appropriation est un discours ; un discours qui raconte les mythes originels, qui décrit les épopées fondatrices et qui inscrit dans la mémoire les grands moments historiques d'un groupe ou d'un territoire. Mais s'approprier, c'est aussi prendre possession de l'espace et prendre position par rapport à l'espace : comment je me positionne par rapport à mon territoire, comment je le vis, je le vois. Une des formes possibles de l'appropriation est la patrimonialisation. Unfinished Italy met en évidence le processus de patrimonialisation qui se développe à partir de ces infrastructures inachevées, processus par lequel un bien est transformé en objet du patrimoine et qui, en tant que tel, fait l'objet de 
mesures de conservation et de restauration. Le patrimoine est donc un construit social (Péron, 2002), un outil au service de la légitimation de la position sociale de certains acteurs (Amougou, 2004) ou au service de l'appropriation d'un territoire par certains groupes sociaux (Veschambre, 2008) : le patrimoine n'existe pas donc a priori (Choay, 1996), même s'il est élaboré à partir de biens existants qui constituent en quelque sorte un patrimoine potentiel. C'est le résultat d'une démarche qui associe divers acteurs selon les contextes (pouvoirs publics, citoyens, institutions, artistes, professionnels, scientifiques, érudits...) et qui se nourrit de différentes formes de savoirs (vernaculaires, professionnels). Un bien transformé en patrimoine, c'est-à-dire reconnu juridiquement comme tel, acquiert donc le statut d'un héritage à transmettre ; c'est un bien qui appartient à l'ensemble de la communauté et qui est censé permettre d'établir un lien entre les générations : une fois institutionnalisé, le patrimoine possède donc une dimension collective et sa conservation relève donc de l'intérêt général.

Produire un patrimoine consiste donc à réinjecter du sens et de la valeur dans un édifice qui, soit, a perdu sa fonction d'origine et dont l'abandon remet en cause sa conservation (situation la plus courante), soit, n'a pas acquis de fonction faute d'avoir été achevé et d'avoir été opérationnel (situation envisagée ici).

Quelle histoire nous raconte la patrimonialisation de l'inachevé ? Comment fonctionne ce discours? Le discours fonctionne sur la base d'une tension entre valorisation et dévalorisation (la valorisation a besoin de son antinomie pour exister). Dévalorisation dans la mesure où ce discours s'attache à retracer l'histoire de ces équipements et, au-delà, les logiques qui ont sous-tendu la politique d'équipement et d'aménagement du territoire en Sicile à l'époque contemporaine (entre 1960 et 2000) : dévoiement de l'intérêt général pour s'enrichir et se construire des positions de pouvoir politique. Valorisation dans la mesure où ce discours s'attache à retourner le stigmate et à faire d'une charge une ressource (pour la construction d'une identité locale, économique via le tourisme) :

- les équipements inachevés symbolisent, dans la mémoire collective, la corruption et la «mauvaise » politique ${ }^{6}$ : signes de l'échec, traces tangibles de magouilles et d'affaires louches, jusqu'à présent ils sont perçus et vécus comme la marque d'une infamie, d'une histoire dont on veut se défaire ;

- ce processus de patrimonialisation retourne le stigmate en mettant en scène une communauté locale qui décide de se réapproprier ces équipements inachevés et laissés à l'abandon (donc à les entretenir), à communiquer à leur propos (donc dire ce qu'ils sont et pourquoi ils sont ainsi) et à les inscrire dans une politique touristique (turismo del incompiuto): le stigmate est brandit comme une fierté pour construire quelque chose/autre chose.

Ainsi, la ville de Giarre dans la région de Catane a fait le choix non seulement de mettre son patrimoine inachevé à disposition de sa population en le rendant accessible et appropriable (travaux de mise en sécurité, débroussaillage, etc.), mais également d'en faire une stratégie de développement économique en ciblant une forme touristique singulière. La mise en scène de l'inachèvement a donné lieu à la tenue d'un festival (Festival Incompiuto

\footnotetext{
${ }^{6}$ Pour qui les réalités italiennes sont familières, on peut être amené à s’interroger pour savoir dans quelle mesure certains équipements publics inachevés n’ont-ils pas, dès leur conception, été conçus pour être inachevés ? Les marchés publics sont en effet des occasions d'enrichissement personnel et de financement illicite des partis politiques, un moyen d'animer des réseaux et de structurer des clientèles, d'alimenter du consensus social et de mettre en scène une forme d'évergétisme public en montrant, par l'ouverture de chantiers, que les autorités se soucient du bien être de leurs administrés.
} 
siciliano) en juillet $2010^{7}$. Au programme : ateliers de travail, assemblées citoyennes, circuits touristiques, concerts, spectacles. Autant de manifestations visant à transformer la ville de Giarre, «élue » capitale de l'inachèvement par la densité d'équipements inachevés rapportés à la population (8 pour 10000 habitants), en un «chantier de planification urbaine participatif » (Fondazione incompiuto siciliano). Ce festival a été précédé par la création du Parc archéologique de l'inachèvement sicilien (Parco archeologico dell'incompiuto siciliano) en novembre 2007 dans le but de «promouvoir le territoire en développant une nouvelle économie liée au tourisme durable ». Le visiteur peut ainsi visiter 12 équipements inachevés parmi lesquels la piscine olympique de $49 \mathrm{~m}$ (il lui manque un mètre pour être homologable), le théâtre municipal dont le financement quasi ininterrompu des travaux a débuté dans les années 1950, un parking public sans sortie (achevé en 2010 avec des fonds privés), un circuit pour la pratique de l'automodélisme ou le stade de polo (les financements publics pour les équipements sportifs «classiques » à l'occasion de l'organisation des jeux interuniversitaires de 1997 étant épuisés, le maire a déposé une demande de crédit pour ce qui « restait » et obtenu ce stade pour ses concitoyens d'une capacité de 22000 spectateurs alors que la ville compte moins de 30000 habitants).

L'inachèvement est ainsi à la base d'un projet collectif et d'un projet de territoire. Le processus d'appropriation conduit à modifier le rapport d'une communauté à son espace. Alors que précédemment, ces équipements inachevés et abandonnés étaient communément ignorés, ne constituaient pas des éléments structurants des espaces de vie, dès lors qu'ils sont mis en scène et valorisés par le processus de patrimonialisation, ils contribuent à la création d'un «nous» (i.e. sentiment d'appartenance collectif) et deviennent un élément d'identification.

\section{Conclusion}

Il est nécessaire de penser l'inachèvement dans sa dynamique et non de le voir comme un état figé, autrement dit d'introduire de la temporalité dans l'étude du phénomène: l'inachèvement renvoie en effet au continu et au discontinu, il remet en cause l'image linéaire (décision, mise en œuvre, évaluation) que l'on a communément de l'action publique. Au travers de ces processus d'appropriation, non réductibles à la patrimonialisation, les constructions inachevées acquièrent une valeur patrimoniale, communautaire, artistique, économique, paysagère après avoir connues un processus d'abandon. Les projets inachevés continuent ainsi d'alimenter un imaginaire, d'influencer des initiatives, d'agir de manière implicite, de susciter des politiques publiques (comme la volonté des autorités italiennes de faire achever les infrastructures publiques inachevées) et de produire des géographies.

Ces initiatives nous invitent à rompre avec une lecture de l'inachèvement qui ne retient que les signes d'une pathologie (la corruption politique) ou d'un dysfonctionnement de l'action publique ; elles nous conduisent à prendre conscience que ces constructions ont désormais une existence à part entière, en dehors du projet qui les a vu naître. L'inachèvement apparaît alors comme une invitation : il appelle le destinataire, le riverain ou l'artiste à agir, à participer et à se mobiliser; non pas tant à achever ce qui ne l'est pas mais à poursuivre, à reprendre sur un autre mode, à s'inscrire dans une continuité (puisque on ne détruit pas...) tout en s'en détachant.

\footnotetext{
${ }^{7}$ Le projet Incompiuto siciliano a fait l'objet d'une présentation à l'occasion de la XII ${ }^{\mathrm{e}}$ biennale d'architecture de Venise : un pilier en béton de la cité des enfants inachevée de Giarre a été publiquement et officiellement scié pour être exposée à la Biennale (http://www.artsblog.it/post/6141/la-colonna-dellincompiuto-siciliano-allabiennale-di-venezia).
} 


\section{Bibliographie :}

AMOUGOU É. (dir.) (2004), La question patrimoniale. De la «patrimonialisation» à l'examen de situations concrètes, Paris, L'Harmattan.

BELLO MARCANO, M. (2013), «Le songe circulaire: pour comprendre les ruines », Sociétés, ${ }^{\circ} 120$, p. 5-11.

BOURNE, L. (1993), « The demise of gentrification? A commentary and prospective view », Urban Geography, vol. 14, n ${ }^{\circ}$, p. 95-107.

CARPENTER J., LEES L. (1995), «Gentrification in New York, London and Paris: An International Comparison », International Journal of Urban and Regional Research, vol. 19, $\mathrm{n}^{\mathrm{o}} 2$, p. 286-303.

CHOAY F. (1996), L'allégorie du patrimoine, Paris, Le Seuil.

CLERVAL A. (2010), «Les dynamiques spatiales de la gentrification à Paris », Cybergeo : European Journal of Geography [En ligne].

DI MEO G. (2007), «Processus de patrimonialisation et construction des territoires », in BOUFFANGE S., MOISDON P. (dir.), Regards sur le patrimoine industriel de PoitouCharentes et d'ailleurs, La Crèche, Geste.

DUPUY C., HALPERN C. (2009), «Les politiques publiques face à leurs protestataires », Revue française de science politique, $\mathrm{n}^{\circ}$ 4, p. 701-722.

FOURNET F. (2007), «Esthétique de la ruine dans l'itinéraire de Paris à Jérusalem de Chateaubriand », L'information littéraire, ${ }^{\circ} 2$, p. 22-32.

FOURNIAU J-M., DE DONZEL A. (2001), «Le conflit du TGV Méditerranée : la structuration d'un espace public de discussion de la légitimité des décisions », in DONZEL A. (dir.), Métropolisation, gouvernance et citoyenneté dans la région urbaine marseillaise, Paris, Maisonneuve et Larose, p. 467-485.

FOURNIAU J.-M. (2007), «L'expérience démocratique des « citoyens en tant que riverains » dans les conflits d'aménagement », Revue européenne des sciences sociales, $\mathrm{n}^{\circ} 136, \mathrm{p} .149$ 179.

GUMUCHIAN H. (1991), Représentations et aménagement du territoire, Paris, Economica. HASSENTEUFEL P. (2011), Sociologie politique : l'action publique, Paris, Armand Colin. LABORIER P., TROM D. (dir.) (2003), Historicités de l'action publique, Paris, PUF.

LEES L., BONDI, L. (1995), «De-gentrification and economic recession: the case of New York City », Urban Geography, vol. 16, n 3, p. 234-253.

LEES L. (2009), «Urban renaissance in an urban recession: the end of gentrification? », Environment and Planning A, vol. 41, $\mathrm{n}^{\mathrm{o}}$ 7, p. 1529-1533.

LEVY J. (1998), Qu'est-ce que le virtuel ?, Paris, La Découverte.

LOLIVE J. (1997), «La montée en généralité pour sortir du Nimby. La mobilisation associative contre le TGV Méditerranée », Politix, n³9, p. 109-130.

MACCAGLIA F. (2013), «Trafic d'influence et privatisation de l'État : le cas de la passation des marchés publics dans la filière déchets en Sicile », Espaces et sociétés, ${ }^{\circ}$ 4, p. 13-35.

MAGNAGHI A. (2003), Le projet local, Spirmont, Mardaga.

MELE P. (2008), «Conflits et controverses: de nouvelles scènes de production territoriale ? », in GARAT I., SECHET R., ZENEIDI D. (dir.), Espaces en (trans)action, sous, Rennes, Presses universitaires de Rennes, p. 239-250.

MELE P. (dir.) (2013), Conflits de proximité et dynamiques urbaines, Rennes, Presses universitaires de Rennes.

MENY Y., THOENIG J.-C. (1989), Politiques publiques, Paris, PUF.

PÉRON F. (dir.) (2002), Le patrimoine maritime. Construire, transmettre, utiliser, symboliser les héritages maritimes européens, Rennes, Presses universitaires de Rennes. 
RIPOLL F., VESCHAMBRE V. (2005), «Introduction. L'appropriation de l'espace comme problématique », Norois, $\mathrm{n}^{\circ} 195$, p. 7-15

RUFAT S. (2008), « Bucarest, l'éternel retour», Géographie et Cultures, n 65, p. 53-72.

SUBRA P. (2007), Géopolitique de l'aménagement du territoire, Paris, Armand Colin.

SMITH N. (1996), The new urban Frontier: Gentrification and the revanchist City, Londres,

Routledge.

SMITH N. (2002), «New Globalism, New Urbanism: Gentrification as Global Urban Strategy », Antipode, vol. 34, n ${ }^{\circ} 3$, p. 427-450.

SMITH N. (2003), «La gentrification généralisée : d'une anomalie locale à la "régénération urbaine » comme stratégie urbaine globale », in BIDOU-ZACHARIASEN C. (dir.), Retours en ville, Paris, Descartes \& Cie, p. 45-72.

SOKOLOFF B. (1999), Barcelone: ou comment refaire une ville, Montréal, Presses de l'université de Montréal.

VESCHAMBRE V. (2005), « le recyclage urbain, entre démolition et patrimonialisation : enjeux d'appropriation symbolique de l'espace », Norois, n 195, p. 79-92.

VESCHAMBRE V. (2008), Traces et mémoires urbaines. Enjeux sociaux de la patrimonialisation et de la démolition, Rennes, Presses universitaires de Rennes.

\section{Manifesto dell'Incompiuto Alterazioni video}

1. L'incompiuto siciliano è il paradigma interpretativo dell'architettura pubblica in Italia dal dopoguerra ad oggi.

2. Le opere incompiute sono rovine della surmodernità, monumenti generati dall'entusiasmo creativo del liberismo.

3. L'incompiuto siciliano si inserisce nel paesaggio in modo incisivo e radicale. Il processo di creazione delle opere pubbliche Incompiute celebra la conquista del paesaggio da parte dell'uomo moderno.

4. Postulato dell'Incompiuto è la parziale esecuzione del progetto e il caratterizzarsi di continue modifiche nel tempo capaci di generare nuove spinte in avanti.

5. La natura, per mezzo della vegetazione spontanea, dialoga sinesteticamente con le opere incompiute riappropriandosi dei luoghi e ridefinendo il paesaggio.

6. Le opere incompiute hanno nel cemento armato il loro materiale costitutivo. I colori e la superficie sono determinati dalla degradazione dei materiali per effetto del tempo e degli agenti naturali.

7. Nell'Incompiuto Siciliano la tensione tra funzione e forma si risolve. Ecco che il difetto dell'uso diviene opera d'arte.

8. L'Incompiuto Siciliano riassembla e raccoglie luoghi metafisici della contemplazione del pensiero e dell'immaginazione.

9. L'Incompiuto è fondato su un'etica e un'estetica propria.

10. L'Incompiuto Siciliano come simbolo del potere politico e della sensibilità artistica.

\section{Manifeste de l'Inachevé Alterazioni vídeo}

1. L'inachevé sicilien est le paradigme interprétatif de l'architecture publique en Italie entre l'après-guerre et nos jours.

2. Les ouvrages inachevés sont des ruines de la surmodernité, des monuments produits par l'enthousiasme créatif du libélalisme.

3. L'inachevé sicilien s'insère dans le paysage de manière incisive et radicale. 
Le processus de création des équipements publics Inachevés célèbrent la conquête du paysage de la part de l'homme moderne.

4. L'Inachevé postule l'exécution partielle du projet et se caractérise par des modifications continuelles dans le temps capables de générer de nouvelles impulsions.

5. La nature, via une végétalisation spontanée, dialogue en synesthésie avec les ouvrages inachevés en se réappropriant les lieux et en redéfinissant le paysage.

6. Les ouvrages inachevés ont dans le béton armé leur matériau constitutif. Les couleurs et la surfasse sont déterminées par la dégradation des matériaux sous l'effet du temps et des agentes naturels.

7. Dans l'Inachevé sicilien la tension entre fonction et forme se résout. C'est ainsi que l'absence d'utilisation devient œuvre d'art.

8. L'Inachevé Sicilien agrège et réunit les lieux métaphysiques de la contemplation de la pensée et de l'imagination.

9. L'Inachevé se fonde sur une éthique et une esthétique qui lui sont propres.

10. L'Inachevé Sicilien comme symbole du pouvoir politique et de la sensibilité artitistique. 